\title{
Predicting the future from the past
}

\author{
Alvar Agustí1,2 \\ Affiliations: ${ }^{1}$ Respiratory Institute, Hospital Clínic, Institut d'Investigacions Biomèdiques August Pi i Sunyer \\ (IDIBAPS), University of Barcelona, Barcelona, Spain. ${ }^{2}$ Centro de Investigación Biomédica en red Enfermedades \\ Respiratorias (CIBERES), Madrid, Spain. \\ Correspondence: Alvar Agustí, Respiratory Institute, Hospital Clínic, Villarroel 170, 08036 Barcelona, Spain. \\ E-mail: AAGUSTIAclinic.cat
}

@ERSpublications

Is it possible to leverage medical advances from the last $\mathbf{4 0}$ years to predict future changes? http://ow.ly/A1zw305H5a5

Cite this article as: Agustí A. Predicting the future from the past. Eur Respir J 2017; 49: 1601854 [https://doi.org/10.1183/13993003.01854-2016].

The future cannot be predicted, but futures can be invented.

Dennis Gabor (Nobel Prize in Physics), 1963

\section{Introduction}

Like everyone else I am getting older, and I am being asked to talk more and more about the future (a coincidence?). By definition, these talks are speculative (there is no evidence for the future) and they reflect, almost exclusively, a personal view, which in turn arises from my own by definition restricted vital experiences. While preparing one of these talks, I thought that nobody would have been able to predict what was about to happen (the future) in the nearly 40 years that followed my graduation from medical school in 1979. Below I list a number of things that have occurred from then until now. To some extent, this is a sort of experiment where, knowing de facto what has occurred, we ask ourselves if we would ever have been able to predict these events (i.e. can we predict the future from the past?). I believe that this exercise illustrates well the difficulties in predicting the future, since the answer to this question is almost certainly no. The list, not meant to be exclusive or prioritised, includes several very significant advances.

\section{Past advances \\ Advances in general \\ Computers}

Computers have changed the way we work and understand the world. Among many other things, computers have allowed the development of internet, e-mail and PubMed; older readers will, no doubt, remember the many hours spent in the library searching the endless Index Medicus, whereas now PubMed is a mouse-click away (figure 1)! Likewise, computers allowed the development of electronic health records; older readers will remember those thick, heavy paper clinical histories of chronic patients with several concomitant diseases! Electronic health records have many positive aspects no doubt [1]; however, a word of caution is needed if we intend to use them for research (so called "Big Data") since many of them may not have the quality required for research purposes [2].

\section{Mobile technologies}

Mobile phones, tablets and the like have offered a personal, wearable connection to the internet that all young residents use to access up-to-date health-related information platforms, as well as other sources,

Received: Sept 202016 | Accepted after revision: Sept 252016

Conflict of interest: Disclosures can be found alongside this article at erj.ersjournals.com

Copyright @ERS 2017 
FIGURE 1 Evolution of medical information from a) the Index Medicus to d) PubMed, facilitated by $b)$ the emergence of computers, which in turn allowed c) the development of the internet.
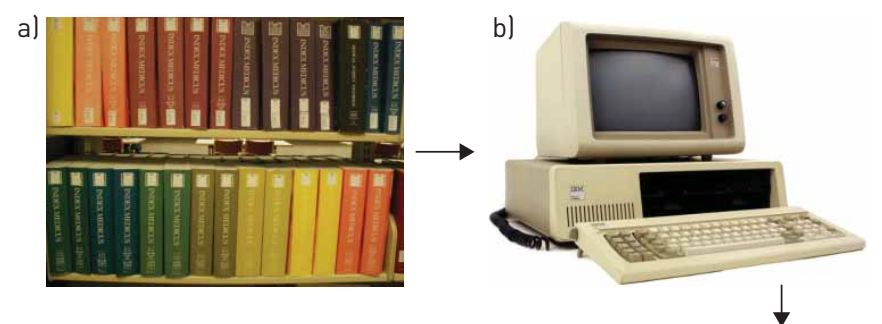

d)

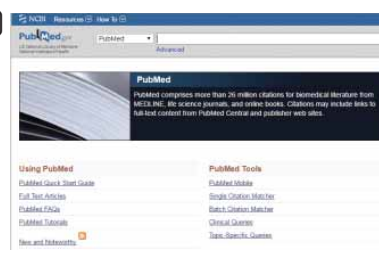

c)

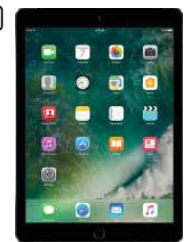

when faced with a clinical problem. In fact, basically all of us have a personal computer in our pocket that is able to make telephone calls besides! This is facilitating tele-health and novel forms of digital research.

\section{Imaging techniques}

Imaging techniques, including echography, computed tomography (CT), magnetic resonance imaging and positron emission tomography, have undergone a sort of big bang that has changed drastically the way many patients are diagnosed and treated.

\section{Minimally invasive procedures}

New procedures including coronary artery stenting, heart-valve replacement using peripheral artery access, laparoscopic and video-assisted thoracic surgery and natural orifice translumenal endoscopic surgery have been developed and implemented in clinical practice.

New diseases

New diseases such as HIV, Ebola and Zika have emerged and had a very significant impact on the population.

\section{New aetiologies (and treatments) for old diseases}

New aetiologies (and treatments) for old diseases have emerged, such as the use of antibiotics to treat Helicobacter Pylori in peptic ulcer cases, which has ended a glorious time for many gastric surgeons!

\section{Bringing Mendel's peas to the clinic}

Mendel's peas (figure 2) used to be a sort of funny historical anecdote in my years at medical school; however, they now without doubt represent the future (even the present?) for the prediction, understanding and tailored treatment of many (if not all) human diseases [5]. It is of note that these peas and the associated Mendelian traits are now much more difficult to understand, since they do not depend only on one or more specific genes but also on many functional factors that modulate their final clinical impact (e.g. epigenetics).

\section{Intensive care units}

Intensive care units (ICUs) were nonexistent when I graduated (at least in Spain). The first examples were (again) led by cardiologists (as coronary units) and, sometime later, more general ICUs began to emerge in hospitals. Monitoring and life-sustaining techniques (ventilators, aortic pumps and other devices) have been instrumental in saving many lives since.

\section{Guidelines}

Guidelines for the most prevalent diseases, based as much as possible in scientific evidence generated via randomised clinical trials (so-called evidence-based medicine), have become prevalent [6].

\section{Advances in respiratory medicine}

Admittedly, some of the advances named above facilitated the emergence of others in a sort of concatenated chain of events. For instance, advances in computer technologies facilitated new imaging techniques, which in turn allowed invasive procedures and monitoring techniques. In addition, this is a list of general medical advances; however, if I look into my own field of medical expertise (respiratory 
a)

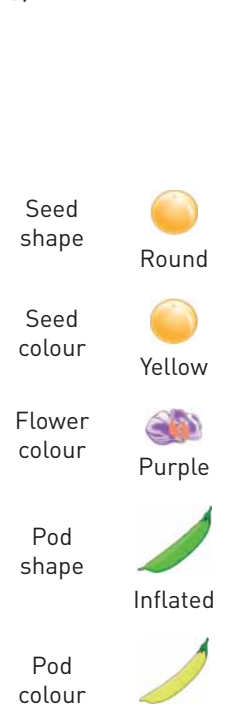

Yellow

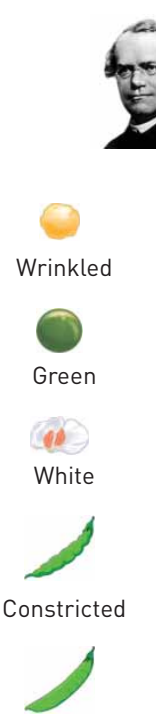

Green

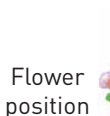

position

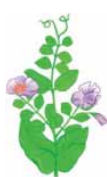

Axial

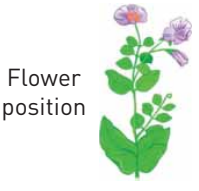

Tall b)

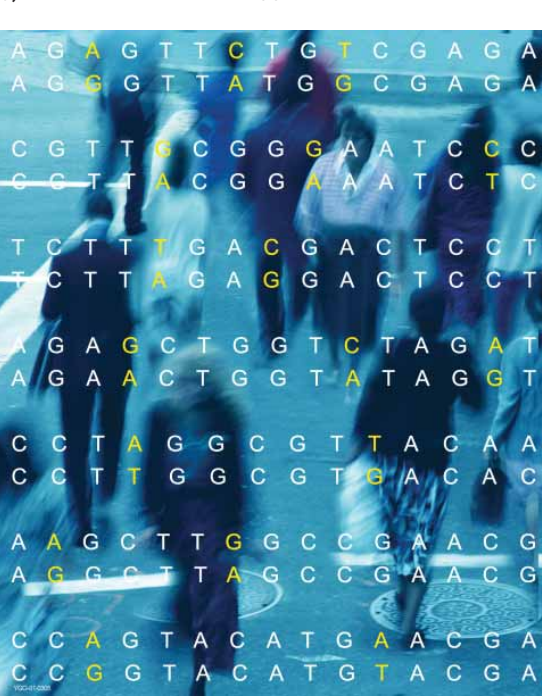

FIGURE 2 The transition from a) Mendel's peas (with the seven traits he studied) to b) the cloning of the human genome, which started the modern post-genomic era. a) The Mendel's peas image was reproduced and modified from [3] with permission b) Reproduced and modified from [4] with permission.

diseases) it is apparent that many advances have also occurred in this field since I graduated in 1979 and most, if not all of them, would have been extremely difficult to predict at that time.

Metered dose inhalers

Metered dose inhalers, later followed by dry powder inhalers, have dramatically changed the way patients with airway diseases are currently treated, for the most part with great success. Inhaled drugs were, initially, short-acting bronchodilators (e.g. salbutamol and ipratropium) [7, 8]. Soon after, inhaled steroids were also introduced and this again changed the asthma landscape dramatically for the better, albeit not completely [9]. More recently, both long-acting $\beta$-agonists and long-acting muscarinic antagonists were introduced and are now combined in single inhalers. By and large this is to the benefit of patients, although some controversies still exist [10].

\section{Pulse oximetry}

Pulse oximetry has allowed the emergence of a new medical field (sleep medicine) and the definition of a new disease, obstructive sleep apnoea [11]. This identification was soon followed by a novel treatment, continuous positive airway pressure [12], which in turn led to the development of noninvasive ventilation, which is now used successfully for the treatment of several acute conditions (such as exacerbations of chronic obstructive pulmonary disease and heart failure) as well as several chronic conditions (such as respiratory failure of skeletal or muscular origin). The latter can be complemented by domiciliary long-term oxygen therapy when needed.

\section{Fibreoptic bronchoscopy}

Nowadays considered a standard, fibreoptic bronchoscopy was a true revolution for the respiratory community, allowing several diagnostic and therapeutic interventions.

\section{Genetic diseases}

Amazing advances have occurred in the understanding of the basic molecular abnormalities and treatment of diseases such as $\alpha_{1}$-antitrypsin deficiency and cystic fibrosis, resulting in dramatic improvements in the life expectancy of patients [13-15]. 
Lung cancer

Screening using low-dose CT has demonstrated a $20 \%$ reduction in mortality in lung cancer cases [18]. In addition, recent achievements in the treatment of lung cancer, including the use of the epidermal growth factor receptor (e.g. rociletinib) [19] and the novel check-point inhibitors (e.g. nivolumab) [20], have improved the survival rate of these patients, albeit that a lot remains to be done in this area.

\section{Pulmonary hypertension}

Traditionally considered only in the context of "cor pulmonale" (i.e. pulmonary hypertension due to a respiratory disease) and/or heart failure, pulmonary hypertension is now recognised more and more as a primary (i.e. idiopathic) disease or as a disorder associated with other systemic disorders or chronic pulmonary emboli [21].

As stated above, this does not pretend to be "the list". I apologise if anyone considers that other important events should have been included, they are likely to be right. This is only a list out of my mind (after giving some thought to it though). In any case, did all these advances occur at no cost? Of course not! All of them required a significant amount of research and investment. Have we forgotten something by having them so at hand? I believe that the answer is yes. I am particularly concerned that young doctors seem to be becoming increasingly techno-dependent and may forget (or worse, may never have been trained in) some very basic aspects of medical care, including a careful and well-integrated medical history and a detailed physical exam. Only after these first two key steps in medical practice have been undertaken should a physician integrate this information and think (always a good thing to do) about the differential diagnosis and the most cost-efficient and safe diagnostic test and/or therapeutic alternatives. Needless to say, excessive technological dependence may also jeopardise the necessary patient-physician human relationship.

\section{Potential future advances}

Considering all of the above, does anyone think that the future, say over the next 40 years, can be predicted accurately? I propose here that we take advantage of modern technologies and start a conversation within the European Respiratory Society (via the European Respiratory Journal (ERJ) website?) where people can predict what will happen. I will open this conversation here. My list below includes some general medical issues but is mostly focused on our common area of expertise: respiratory health. Again, it does not pretend to be "the list" and is not prioritised. I believe that the following will occur in the next 40 years and have categorised the topics as highly likely (we are almost there) and unpredictable (but possible; if not, I should have left this space blank!). Please join me in this conversation.

\section{Highly likely advances \\ Precision medicine [22]}

Most human diseases are heterogeneous. More and more, their appropriate prevention and treatment will require a precision diagnosis (e.g. biomarkers) and a precision treatment (e.g. biologics) targeting specific endotypes (not symptoms at large, as we do now in many cases) [23]. This implies moving from population-derived risk factors (i.e. randomised clinical trials generating evidence-based medicine) to individual specific ones (how do we do this?).

\section{New classifications of disease [24, 25]}

The current classification of diseases is becoming out-of-date in this post-genomic era because it includes different disease states under the same diagnostic category. It does not generally consider individual susceptibility to disease and it cannot be used to individualise modern biological therapies [24, 26]. We should be (and will be) more interested in understanding the biology underlying different diseases than in arguing about the most appropriate diagnostic label, as we often do today [22, 25].

\section{The microbiome}

We humans live in close contact with a myriad of bacteria, viruses and fungi, not only in the skin or gut (as we were traditionally taught) but also in other organs, such as the lungs. The lungs were traditionally thought to be sterile in a healthy subject simply because the traditional microbiological culture techniques were not able to facilitate the growth of all these species and were, therefore, considered negative. By contrast, the use of modern molecular techniques has rapidly destroyed this dogma; however, we do not yet know how these communities of different micro-organisms in the lungs (and elsewhere) interact with the epithelium both in health and disease, but this is likely to be extremely important. Furthermore, the gut is likely to become a new immune organ, so we will have to pay more attention to the gut microbiome in our respiratory patients. 


\section{Beyond smoking}

Smoking is, no doubt, a major respiratory insult but, more and more, other environmental stressors (besides infectious agents) such as air pollution and microparticles will be considered more carefully in the pathobiology of several respiratory diseases.

\section{Early life events}

Today, an infant with respiratory problems (e.g. wheezing) is very likely to be diagnosed with asthma, yet it is highly likely that due to a variety of genetic and environmental factors the lungs might not develop properly in some individuals. These developmental problems may, at first, lead to a misdiagnosis of asthma in infancy but also to chronic airway diseases in late adulthood [27-30]. Understanding the biological basis of these disorders of lung maturation and identifying these individuals early in life will be important in order to treat them and to prevent later comorbidities.

\section{Earlier lung cancer diagnosis}

By refining the population to screen and/or by combining it with circulating (i.e. liquid biopsies), urinary and/or exhaled biomarkers earlier diagnosis will become possible (so lung cancer can be cured!) [31].

Interventional pulmonology

Pulmonologists will follow the lead of the cardiologists and will become more interventional, although I admit that this is probably more a wish than a prediction!

Space travel

Space travel will require respiratory support and new research.

Social debate on the ethics and sustainability of prolonging life

Is there any limit? Can we sustain any goal? In relation to this, it is likely that healthcare systems will undergo profound organisational changes and that several roles will change (specialised nurses, technologists, etc.).

\section{Smaller (atomic) or integrative (network) medicine}

For the past 400 years, medical research has chosen to go smaller (from the whole body, to organs, to cells, to molecules). What is to be the next step, going even smaller (atomic medicine)? I believe that the next step is to be more integrative. Biology is about networks of genes, proteins, lipids, cells, organs and environmental factors, as illustrated in figure 3 [32]. When we were going smaller we used to focus on a progressively smaller number of cells or molecules and, by doing so, we lost track of the general picture (we "couldn't see the wood for the trees!"). More and more, we are trying to integrate available information (e.g. network analysis [33]) and to develop mathematical models of disease (i.e. systems biology [34]) to better understand disease pathogenesis and, by doing so, identify novel therapeutic targets that act in specific areas of the network [35]. This will eventually allow the in silico design of new drugs (synthetic biology [36]).

\section{Global health}

Most of the predictions above refer to the so-called first (or developed) world. Yet, it represents only a minority of the world, where developing countries (or the third world) represent the vast majority. The relationship between both worlds is bidirectional and will become more so. Medicine will probably evolve in developing countries by adopting many of the advances that developed countries already enjoy. However, the third world may also influence the first via a number of mechanisms that include massive immigration and imported old (e.g. tuberculosis) and new (viral?) diseases. Needless to say, global warming may also have significant effects on human health, both in the first and third worlds.

\section{Unpredictable advances}

Prevent and cure all human disease

This is the Holy Grail and the ultimate goal of medicine. To this end, many people in healthcare systems, the drug industry and other health-related companies, as well as in Government, invest a lot of time, effort, creativity and money to improve the treatment of disease. Perhaps, we should change this strategy slightly and try to prevent the occurrence of disease or, at least, diagnose them as early as possible in order to maximise the chances of a complete cure (by the use of wearable, personal biomonitoring devices?). Imagine if, one way or another, in the long-distant future we achieve this goal. If so, it is predicted that humans may live for 150-200 years on average. Would that society be sustainable? If, as most of you might be thinking, the answer is no, why do we keep pursuing this goal? Perhaps some social debate is needed here. 


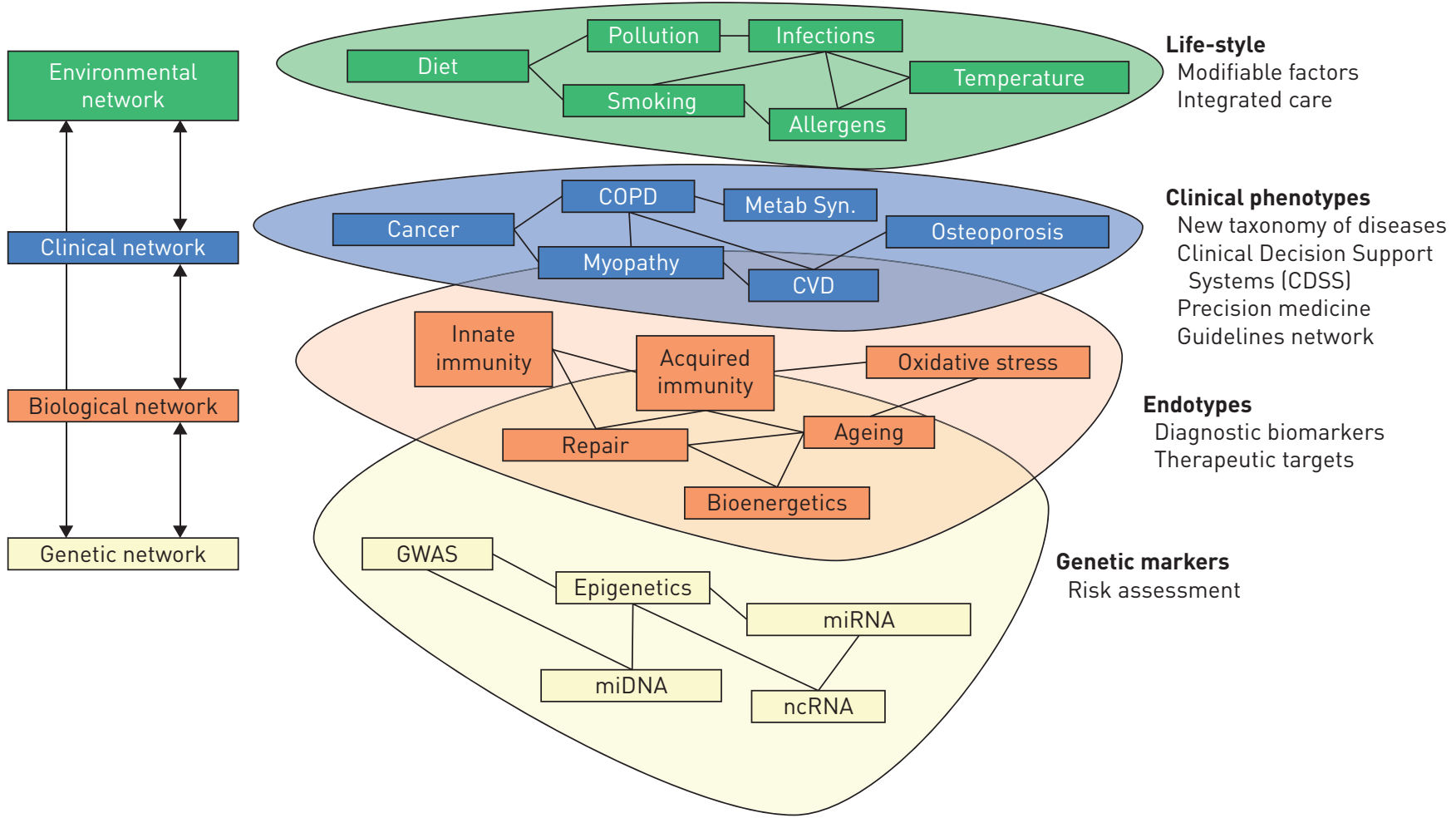

FIGURE 3 A holistic and integrated view of disease from the standpoint of multilevel networks. The figure illustrates the different levels of complexity of chronic obstructive pulmonary disease (COPD), although these are, by and large, applicable to many other chronic diseases. The outcomes of potential clinical relevance are shown in the right-hand column. At each level, only some of the potential components are shown to illustrate the concept (the figure is not intended to be comprehensive). Likewise, links between the different elements of the network are drawn for illustrative purposes only and do not necessarily reflect evidence-based relationships. Metab Syn.: metabolic syndrome; CVD: cardiovascular disease; GWAS: genome-wide association studies; miDNA: mitochondrial DNA; miRNA: micro-RNA; ncRNA: noncoding RNA. Reproduced and modified from [32] with permission.

\section{Organ regeneration}

The field of stem cell research is extremely active today; however, to date, it has not really delivered clinically applicable therapeutics. I hope that it will do so in the future.

\section{Artificial (bioengineered) organs}

As for organ regeneration, the development of bioengineered organs is conceivable; however, it is not clear when this will be a reality, if it eventually is.

\section{Cell and gene therapy}

Both cell and gene therapy have already been around for some time but without too much success so far.

\section{Use of nanoparticles for diagnostic and therapeutic purposes}

This is a very active research field; however, real practical applications are, to my knowledge, still outside daily practice. The potential of this area is huge, particularly if combined with new noninvasive imaging techniques.

\section{Summary}

This is my wish list which, almost certainly, time will prove wrong. Please help me to improve it by participating in the ERJ conversation, which will be open for 3 months. After that time, I will analyse the information and get back to you. If successful (this is up to you, but thanks for participating anyway), such information will be extremely helpful, at least for my next talk on the future!

\section{Acknowledgements}

I thank Rosa Faner, Guillaume Noell and Paco Pozo for critical reading and helpful suggestions and the high-speed train from Madrid to Barcelona that gave me enough time and a quiet atmosphere to think about the content of this paper. 


\section{References}

1 Auffray C, Balling R, Barroso I, et al. Making sense of big data in health research: towards an EU action plan. Genome Med 2016; 8: 71.

2 Reddel HK, Gerhardsson de Verdier M, Agusti A, et al. P138 An innovative approach to study design: using electronic medical records to inform the feasibility and design of the novelty study (a novel observational longitudinal study on patients with asthma and/or COPD). Thorax 2016; 71: Suppl. 3, A157-A158.

3 OpenStax CNX. Mendel's Experiments. http://cnx.org/contents/16db03ea-f31d-48c4-88b9-aa2acb49412e@3http:// cnx.org/contents/16db03ea-f31d-48c4-88b9-aa2acb49412e@3 Date last updated: March 21, 2013.

4 US Dept of Energy. Genomic Science Program. http://genomicscience.energy.gov Image available from https:// public.ornl.gov/site/gallery/detail.cfm?id=417\&topic=46\&citation=\&general=\&restsection .

5 Venter JC, Adams MD, Myers EW, et al. The sequence of the human genome. Science 2001; 291: 1304-1351.

6 Evidence-based medicine. A new approach to teaching the practice of medicine. JAMA 1992; 268: 2420-2425.

7 Chapman KR, Kesten S, Szalai JP. Regular vs as-needed inhaled salbutamol in asthma control. Lancet 1994; 343: 1379-1382.

8 Moayyedi P, Congleton J, Page RL, et al. Comparison of nebulised salbutamol and ipratropium bromide with salbutamol alone in the treatment of chronic obstructive pulmonary disease. Thorax 1995; 50: 834-837.

$9 \quad$ Barnes PJ. Inhaled glucocorticoids for asthma. N Engl J Med 1995; 332: 868-875.

10 Singh D, Roche N, Halpin D, et al. Current controversies in the pharmacological treatment of chronic obstructive pulmonary disease. Am J Respir Crit Care Med 2016; 194: 541-549.

11 Partinen M, Jamieson A, Guilleminault C. Long-term outcome for obstructive sleep apnea syndrome patients. Mortality Chest 1988; 94: 1200-1204.

12 Sullivan CE, Berthon-Jones M, Issa FG, et al. Reversal of obstructive sleep apnoea by continuous positive airway pressure applied through the nares. Lancet 1981; 1: 862-865.

13 Chapman KR, Burdon JGW, Piitulainen E, et al. Intravenous augmentation treatment and lung density in severe $\alpha 1$ antitrypsin deficiency (RAPID): a randomised, double-blind, placebo-controlled trial. Lancet 2015; 386 : 360-368.

14 Stoltz DA, Meyerholz DK, Welsh MJ. Origins of cystic fibrosis lung disease. N Engl J Med 2015; 372: 351-362.

15 Boyle MP, Bell SC, Konstan MW, et al. A CFTR corrector (lumacaftor) and a CFTR potentiator (ivacaftor) for treatment of patients with cystic fibrosis who have a phe508del CFTR mutation: a phase 2 randomised controlled trial. Lancet Respir Med 2014; 2: 527-538.

16 Noble PW, Albera C, Bradford WZ, et al. Pirfenidone for idiopathic pulmonary fibrosis: analysis of pooled data from three multinational phase 3 trials. Eur Respir J 2016; 47: 243-253.

17 Raghu G, Selman M. Nintedanib and pirfenidone. New antifibrotic treatments indicated for idiopathic pulmonary fibrosis offer hopes and raises questions. Am J Respir Crit Care Med 2015; 191: 252-254.

18 Gould MK. Lung-cancer screening with low-dose computed tomography. N Engl J Med 2014; 371: 1813-1820.

19 Sequist LV, Soria JC, Goldman JW, et al. Rociletinib in EGFR-mutated non-small-cell lung cancer. N Engl J Med 2015; 372: 1700-1709.

20 Brahmer J, Reckamp KL, Baas P, et al. Nivolumab versus Docetaxel in advanced squamous-cell non-small-cell lung cancer. N Engl J Med 2015; 373: 123-135.

21 Galie N, Humbert M, Vachiery JL, et al. 2015 ESC/ERS Guidelines for the diagnosis and treatment of pulmonary hypertension. Eur Respir J 2015; 46: 903-975.

22 Agusti A, Bel E, Thomas $\mathrm{M}$, et al. Treatable traits: toward precision medicine of chronic airway diseases. Eur Respir J 2016; 47: 410-419.

23 Woodruff PG, Agusti A, Roche N, et al. Current concepts in targeting chronic obstructive pulmonary disease pharmacotherapy: making progress towards personalised management. Lancet 2015; 385: 1789-1798.

24 Loscalzo J, Kohane I, Barabasi AL. Human disease classification in the postgenomic era: a complex systems approach to human pathobiology. Mol Syst Biol 2007; 3: 124.

25 Sterk PJ. Chronic diseases like asthma and COPD: do they truly exist? Eur Respir J 2016; 47: 359-361.

26 Cesario A, Auffray C, Agusti A, et al. A systems medicine clinical platform for understanding and managing non-communicable diseases. Curr Pharm Des 2014; 20: 5945-5956.

27 Carraro S, Scheltema N, Bont L, et al. Early-life origins of chronic respiratory diseases: understanding and promoting healthy ageing. Eur Respir J 2014; 44: 1682-1696.

28 Lange P, Celli B, Agusti A, et al. Lung-function trajectories leading to chronic obstructive pulmonary disease. N Engl J Med 2015; 373: 111-122.

29 Martinez FD. Early-life origins of chronic obstructive pulmonary disease. N Engl J Med 2016; 375: 871-878

30 Chacko A, Carpenter DO, Callaway L, et al. Early-life risk factors for chronic nonrespiratory diseases. Eur Respir J 2015; 45: 244-259.

31 Molina R, Marrades RM, Auge JM, et al. Assessment of a combined panel of six serum tumor markers for lung cancer. Am J Respir Crit Care Med 2016; 193: 427-437.

32 Agusti A, Vestbo J. Current controversies and future perspectives in chronic obstructive pulmonary disease. Am J Respir Crit Care Med 2011; 184: 507-513.

33 Diez D, Agusti A, Wheelock CE. Network analysis in the investigation of chronic respiratory diseases: from basics to application. Am J Respir Crit Care Med 2014; 190: 981-988.

34 Barabasi AL, Gulbahce N, Loscalzo J. Network medicine: a network-based approach to human disease. Nat Rev Genet 2011; 12: 56-68.

35 Menche J, Sharma A, Kitsak M, et al. Disease networks. Uncovering disease-disease relationships through the incomplete interactome. Science 2015; 347: 1257601.

36 Ruder WC, Lu T, Collins JJ. Synthetic biology moving into the clinic. Science 2011; 333: 1248-1252. 